\title{
Postductal Origin of the Left Carotid, Left Subclavian, and Aberrant Retroesophageal Right Innominate Arteries in Truncus Arteriosus with Interrupted Aortic Arch
}

\author{
T. Ishizaka, ${ }^{1}$ S.W. Allen, ${ }^{2}$ P.J. Strouse, ${ }^{3}$ R.G. Ohye ${ }^{1}$ \\ ${ }^{1}$ Division of Pediatric Cardiovascular Surgery, Section of Cardiac Surgery, C.S. Mott Children's Hospital, \\ University of Michigan Health System, Ann Arbor, MI 48109, USA \\ ${ }^{2}$ Pediatric Cardiology, The Wichita Clinic, Wichita, KS, USA \\ ${ }^{3}$ Section of Pediatric Radiology, C.S. Mott Children's Hospital, University of Michigan Health System, Ann Arbor, \\ MI 48109, USA
}

\begin{abstract}
A neonate presented to the C.S. Mott Children's Hospital at the University of Michigan with truncus arteriosus and interrupted left aortic arch, with associated postductal origin of the left carotid, left subclavian, and aberrant retroesophageal right innominate arteries. In addition, the patient was diagnosed with DiGeorge syndrome. This unique anomaly has not been previously reported. The anatomy, pathophysiology, embryology, and successful surgical management of this anomaly are reviewed in this report.
\end{abstract}

Keywords: Truncus arteriosus — Interrupted aortic arch - Congenital heart disease left subclavian, and aberrant retroesophageal right innominate arteries from the proximal descending aorta (Fig. 2).

Complete repair was undertaken at 18 days of age. The repair was performed through a standard median sternotomy. No thymus gland was present. The left carotid and left subclavian arteries arose postductally from the descending aorta. A postductal aberrant right innominate artery coursed posterior to the esophagus. The single coronary artery arose anteriorly and rightward, giving rise to the right coronary, left anterior descending, and circumflex arteries (Fig. 3). The patient was cooled to $18^{\circ}$ on cardiopulmonary bypass using a single arterial cannula placed into the distal truncus with the branch pulmonary arteries occluded. After aortic cross clamping, a right ventriculotomy was performed inferior and rightward to the left anterior descending coronary artery. The ventricular septal defect was located in the typical position immediately beneath the truncal valve and was closed with a Gore-Tex patch. Aortic arch reconstruction was then performed under deep hypothermic circulatory arrest. The

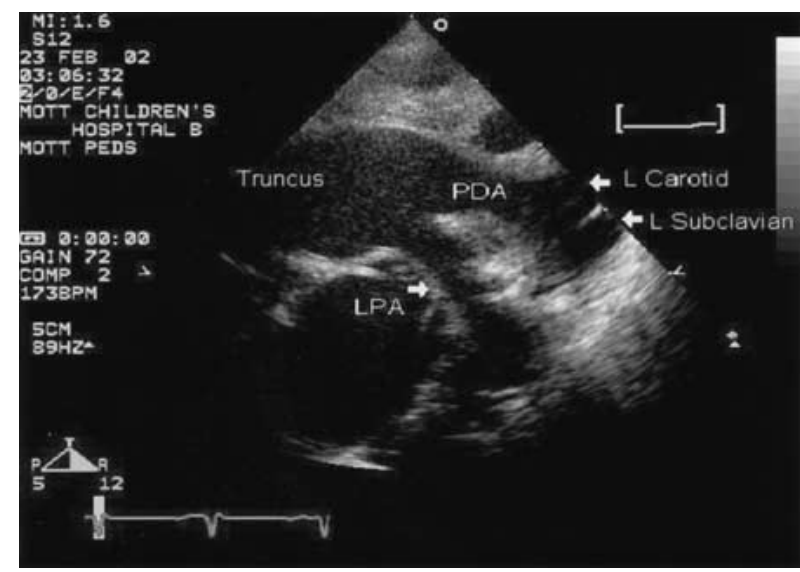

Fig. 1. Parasternal long-axis view of the truncus demonstrating the left pulmonary artery $(L P A)$, patent ductus arteriosus $(P D A)$, and postductal origins of the left carotidand left subclavian arteries.
A $3.0-\mathrm{kg}$ female term infant was noted to be cyanotic with an associated heart murmur and poor perfusion at 24 hours of life. A venous blood gas demonstrated $\mathrm{pH}$ of $6.99, \mathrm{pCO}_{2}$ of $26 \mathrm{mmHg}$, and $\mathrm{pO}_{2}$ of $25 \mathrm{mmHg}$. The patient was intubated and an infusion of prostaglandin $\mathrm{E}_{1}$ was begun. Persistently low ionized calcium levels were noted. Echocardiography demonstrated truncus arteriosus with separate origins of the branch pulmonary arteries (type II) and an interrupted aortic arch. Postductal left carotid and subclavian arteries were identified (Fig. 1). The truncal valve was tricuspid with minimal stenosis or insufficiency. A single coronary artery originated from the right coronary cusp, giving rise to both the right and left coronary arteries. Because the origin of the innominate artery was not well imaged by echocardiography, magnetic resonance imaging with three-dimensional $(3 \mathrm{D})$ reconstruction was performed and demonstrated postductal origin of the left carotid,

Correspondence to: R.G. Ohye, F7830 Mott/0223, 1500 East Medical Center Drive, Ann Arbor, MI 48109, USA. email: ohye@umich.edu 

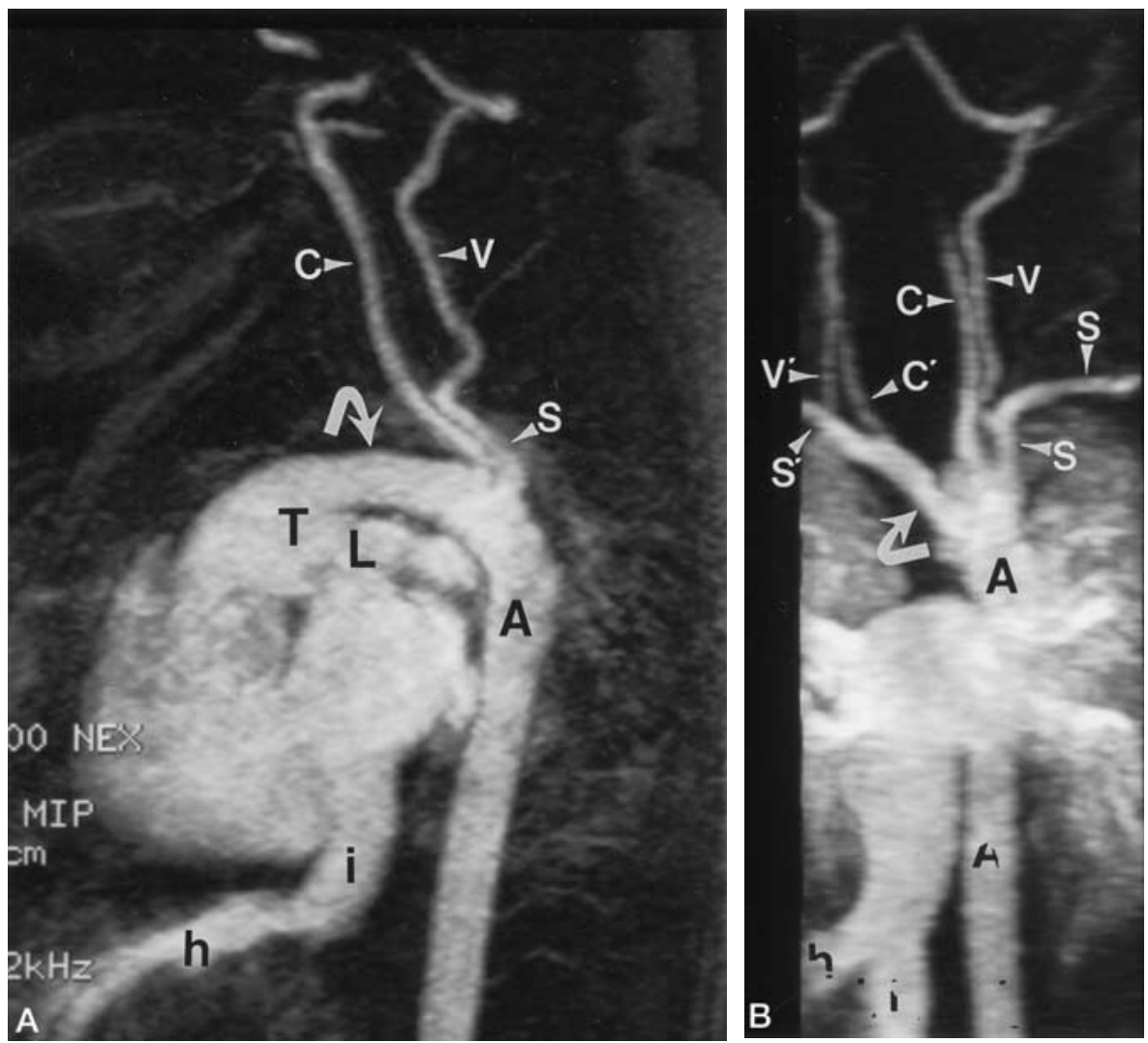

Fig. 2. Maximum intensity projection $(M I P)$ images from a gadolinium-enhanced 3D MR angiogram (TR, $6.1 \mathrm{msec}$; TE, $1.4 \mathrm{msec}$; flip angle, $35^{\circ}, 2$-mm-thick sagittal source images). (A) The sagittal oblique image shows the truncus arteriosus $(T)$ with continuation as a ductal arch (curved arrow). A portion of left pulmonary $\operatorname{artery}(L)$ is seen inferior to the ductal arch. The left common carotid artery $(C)$ and left subclavian artery $(S)$ are seen originating postductally from the cephalad aspect of the arch. $A$, descending aorta-; $i$, inferior vena cava; $h$, hepatic vein; $V$, left vertebral artery. (B) The coronal oblique image shows the innominate artery (curved arrow) originating from the right side of the proximal descending aorta $(A)$. The innominate artery divides into right common carotid artery $\left(C^{\prime}\right)$ and right subclavian artery $\left(S^{\prime}\right)$. The right vertebral artery $\left(V^{\prime}\right)$ originates from the right subclavian artery.

\section{Carotid}

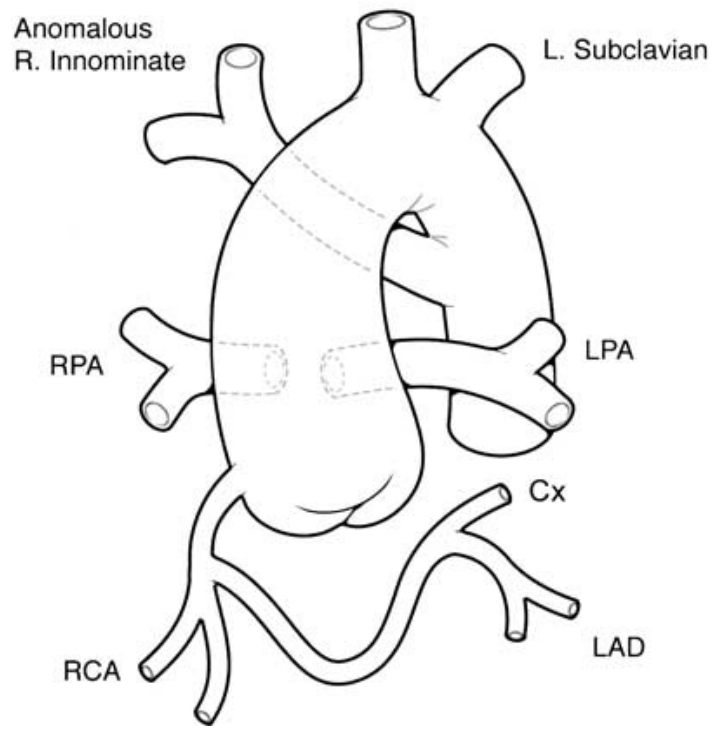

Fig. 3. Truncus arteriosus (type II) with interrupted aortic arch and postductal origin of the left carotid, left subclavian, and anomalous right innominate arteries. A single coronary supplies theright coronary $(R C A)$, left anterior descending $(L A D)$, and circumflex $(C x)$ arteries. $L P A$, left pulmonary artery; $R P A$, right pulmonary artery. ductus arteriosus was divided just distal to the branch pulmonary arteries, and all apparent ductal tissue was resected (Fig. 4 inset). The pulmonary arteries were then excised together with a patch of adjacent truncal wall, and they were transferred anterior to the truncus by a Lecompte maneuver (Fig. 4A). The proximal descending aorta was extensively mobilized, facilitating a direct, tension-free anastomosis between the anterior wall of the truncus and the proximal descending aorta (Fig. 4B). A patch of homograft was tailored and used to augment both the proximal descending aorta and the posterior wall of the truncus where the pulmonary arteries had been harvested (Fig. 4C). An 11-mm cryopreserved pulmonary homograft, augmented by a hood of Gore-Tex patch proximally, was placed between the ventriculotomy and the distal pulmonary arteries (Fig. 5).

The patient was weaned from cardiopulmonary bypass uneventfully and remained hemodynamically stable on minimal inotropic support. The chest was left open to avoid compression of the anterior right ventricle to pulmonary artery conduit until postoperative day 4 . The postoperative course was complicated by persistent atelectasis of the left upper lobe. A mild degree of stenosis of the left main bronchus was identified by bronchoscopy and computed tomography scan and was related to the aortic reconstruction. The patient was treated with expectant observation and was successfully extubated on postoperative day 21 . She was subsequently discharged in good condition. Postoperative echocardiography demonstrated good biventricular function with normal chamber dimensions and a widely patent aortic anastomosis. The extracardiac conduit showed trivial insufficiency and low-velocity laminar flow. 


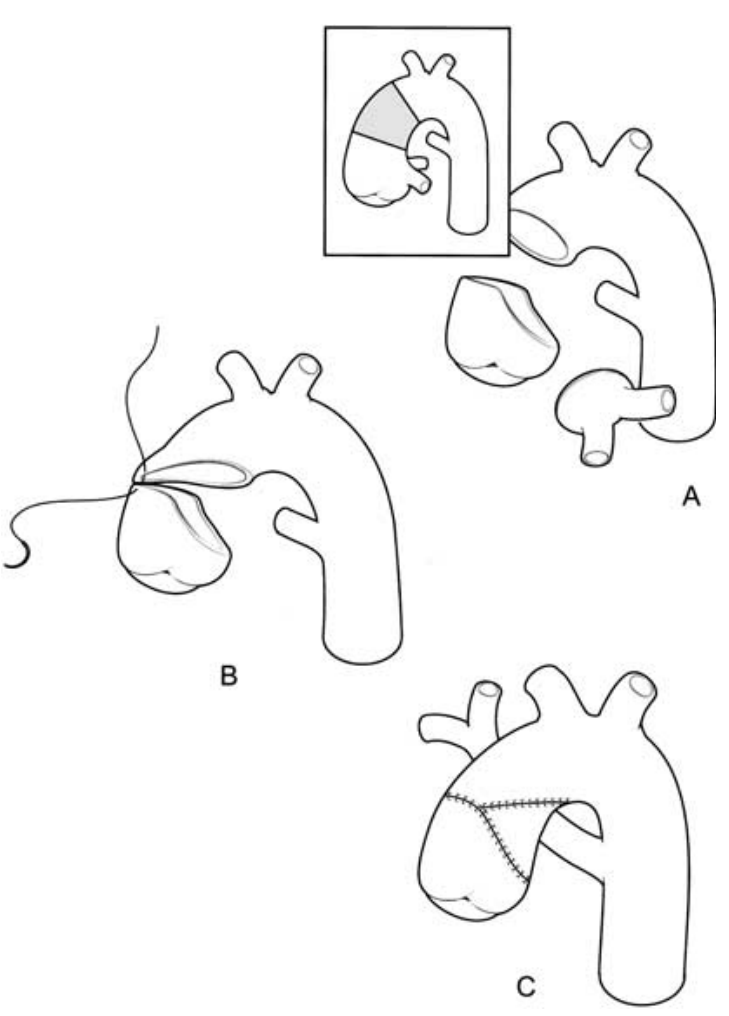

Fig. 4. (Inset). Lateral view of the pre-repair anatomy with the area of the ductus shaded. (A) Lateral view after resection of the ductus and removal of the pulmonary artery button from the truncus. (B) The anterior walls of the truncus and distal aorta are approximated. (C) The posterior aspect of the aortic reconstruction and area of removal of the pulmonary arteries are augmented with a patch of pulmonary allograft.

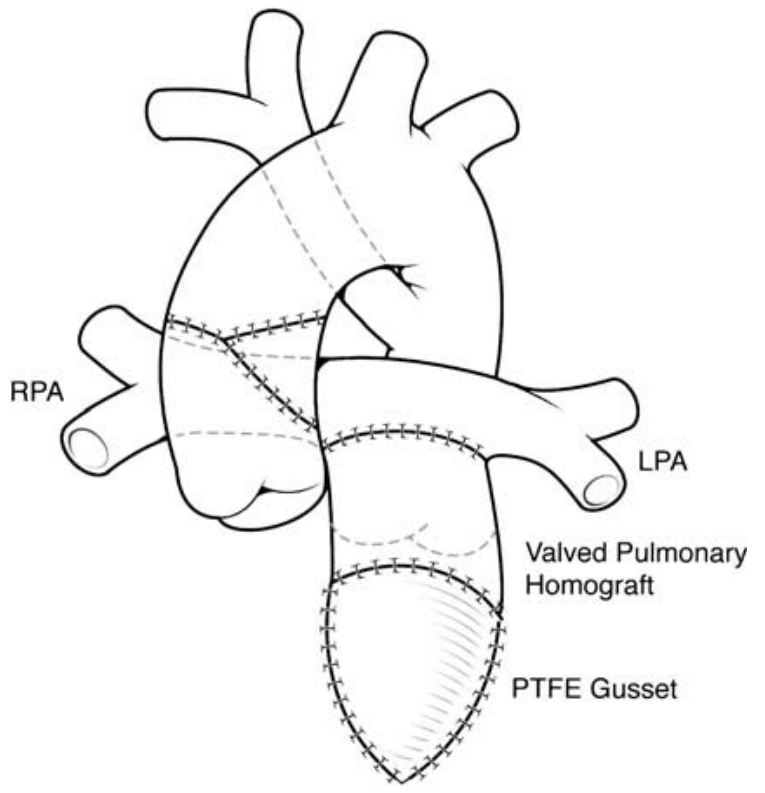

Fig. 5. Anterior view of the completed repair with a pulmonary allograft right ventricle-to-pulmonary artery conduit. $L P A$, left pulmonary artery; $P T F E$, polytetrafluoroethylene; $R P A$, right pulmonary.

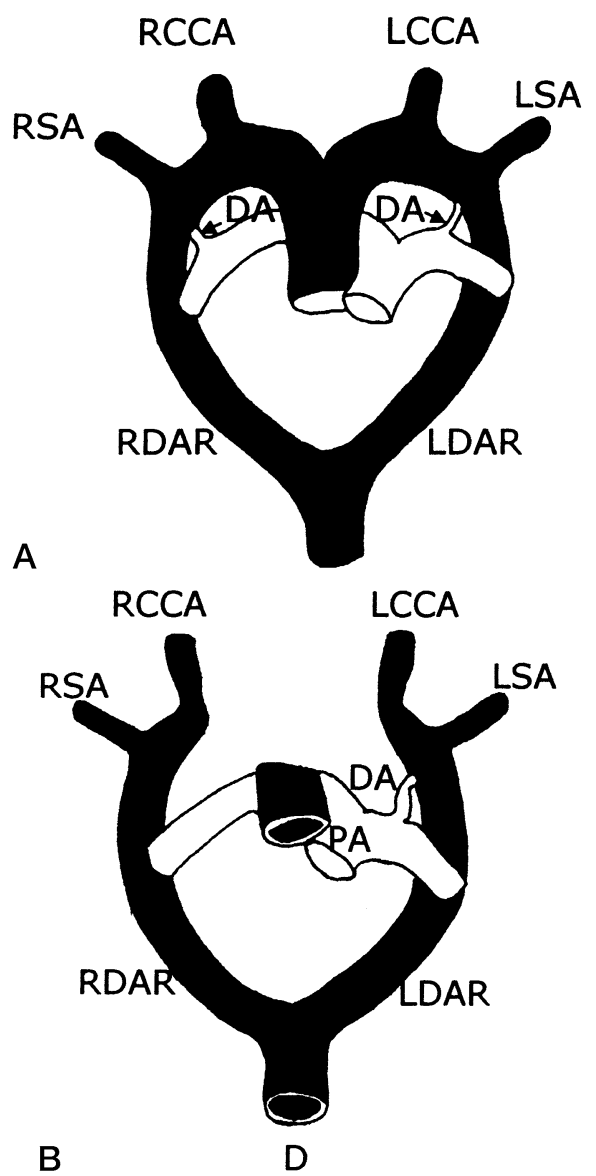

Fig. 6. (A) Edward's embryologic double aortic arch. $R D A R$, right dorsal aortic root; $L D A R$, left dorsal aortic root; $R S A$, right subclavian artery; $L S A$, left subclavian artery; $R C C A$, right common carotid artery; $L C C A$, left common carotid artery; $D A$, ductus arteriosus. (B) Formation of a left interrupted aortic arch with postductal origin of left carotid, left subclavian, and right aberrant

\section{Discussion}

The association of truncus arteriosus with interrupted aortic arch is a rare complex congenital anomaly and represents a surgical challenge [1, 4]. This patient's anatomy was further complicated by an arch interruption proximal to the left carotid artery as well as an aberrant retroesophageal right innominate artery. The combination of these rare anomalies resulted in the postductal origin of all arch vessels from the proximal descending aorta. Although this anomaly has not been previously reported, its occurrence can be explained by the embryologic double aortic arch described by Edwards [2], who described the various aortic arch abnormalities by selective regression of various parts of either arch. The formation of the arch anomaly in our case is assumed to result from the regression of both sides of the aortic arch from 
the point of origin to both common carotid arteries (Fig. 6). In this case, magnetic resonance imaging was useful in defining the vascular anatomy, precluding the need for cardiac catheterization [3]. In repair of truncus arteriosus and interrupted aortic arch, it is generally possible to maintain native tissue continuity and the potential for growth by constructing an anastomosis between the distal truncus and the proximal descending aorta with or without a Lecompte maneuver [1]. In our patient, with no bracheocephalic vessels and arch proximally to extend the length of the truncus, the distance to the descending aorta was greater than generally seen in patients with a typical type B interruption. This most likely contributed to the mild left main stem compression after repair. The development of truncal valve insufficiency and/or failure of growth of the neoaortic arch, despite continuity of native tissue, remain potential late concerns. Use of the Lecompte maneuver not only aided in aortic arch reconstruction, but also should facilitate future right ventricle to pulmonary artery conduit replacements. Late occurrence of dysphagia lusoria from the retroesophageal aberrant right innominate artery is another theoretical, although unlikely, concern.

\section{References}

1. Bove EL, Mosca RS (1995) Lessons learned in truncus arteriosus surgery. Adv Cardiac Surg 6:91-101

2. Edwards JE (1948) Anomalies of derivatives of aortic arch systems. Med Clin North Am 19:925-949

3. Masui T, Katayama M, Kobayashi S, et al. (2000) Gadoliniumenhanced MR angiography in the evaluation of congenital cardiovascular disease pre- and postoperative states in infants and children. J Magn Reson Imaging 12:1034-1042

4. Sano S, Brawn WJ, Mee RBB (1990) Repair of truncus arteriosus and interrupted aortic arch. J Cardiac Surg 5:157162 